\title{
Vaccination and Risk identification \& Risk management: a true controversy?
}

\author{
Jos Noordhuizen* \\ Former Diplomate at EC BHM and VPM, School of Agriculture and Veterinary Science, Charles Sturt University, Boorooma Street, North Wagga, Australia
}

\begin{abstract}
In this short paper two aspects of veterinary preventive medicine are addressed: applying vaccines and implementing risk identification and risk management plans. Both should go hand-in-hand on animal production farms because they are mutually beneficial. Moreover, the potential risk of economic losses is drastically lowered.

In this short paper both domains are briefly highlighted. A dairy farm is used for illustration purposes.
\end{abstract}

\section{Introduction}

Animal production these days cannot function without vaccines. The most important representatives are the more or less intensive poultry and pig farms around the world and to a lesser extent cattle production. Vaccination plans are part of veterinary preventive medicine and cover a wide spectrum of micro-organisms, such as viruses, bacteria, and endo- or ectoparasites.

Preventive medicine, however, should also cover risk identification and risk management. This is a highly relevant domain given that most diseases and disorders in, for example, dairy cattle herds, have a multifactorial background. In this background, risk factors from the cows 'environment such as housing, barn climate, feed, and at the cow level such as the level of genetic disease resistance, metabolism, age, milk production level may play a substantial role in the occurrence of these diseases [1,2].

Veterinary preventive medicine in practice should address both domains: the animal health promotion through vaccination and through risk identification \& management.

This short paper addresses both domains, with the example of dairy cattle for illustration purposes.

\section{Veterinary preventive medicine -- Vaccines}

In the course of a veterinary herd health program for dairy farms the veterinarian addresses different domains of dairy farming, like reproductive performance, milk production \& nutrition, and animal health. Part of the latter is the design of vaccination plans, which may cover a broad spectrum of micro-organisms.

For example, for dairy cattle farms such plans may comprise, starting at young calf level and related to the age of the calves, the different vaccines as named in Table 1.

The contents of a vaccination plan depend on the farmer and the veterinarian. A farmer may assess the economic losses of a certain disease and calculate the costs-benefits of vaccination against this disease. The outcome may force him to ask the veterinarian for such a vaccination plan. On the other hand, even when the farmer does not ask for a vaccination plan, the veterinarian may esteem such a plan beneficial for the farmer and he discusses his proposal with the farmer. Yet another possibility is that a farmer asks for such a plan, while the veterinarian is not truly convinced that this farmer would really need it. It is the field of potential tension between farmer and veterinarian where professional communication plays a key role [3,4]. After all, it is the farmer who decides.

It would be highly advisable that a veterinarian checks what the results of vaccination are using parameters such as monthly and yearly disease incidence, milk production level, the occurrence of other -not vaccine-related-disorders, total costs-benefits assessments. Unfortunately, this is not a common rule everywhere and, hence, there is room for improvement.

\section{Veterinary preventive medicine -- Risk identification and risk management}

As stated earlier, risk identification and risk management are the second domain of veterinary preventive medicine. In the author's experience this domain is largely neglected in the veterinary and dairy world. In fact, this domain should be part of any dairy herd health program, irrespective of whether vaccines are being applied or not.

The reason is simple: by implementing risk management plans within a dairy herd health program, the operational level of the latter is complemented by the first, more tactical in nature. Given the multifactorial nature of animal disease, implicating many contributory risk factors, this approach is crucial (Table 2).

At the same time, implementing risk management plans is far less costly than vaccination and yields results. An illustration of the

${ }^{\star}$ Correspondence to: Jos Noordhuizen, DVM, $\mathrm{PhD}$, Former Diplomate at EC BHM and VPM, School of Agriculture and Veterinary Science, Charles Sturt University, Boorooma Street, North Wagga, NSW, Australia, E-mail: josnoord@ gmail.com

Received: January 06, 2021; Accepted: January 22, 2021; Published: January 25, 2021 
Table 1. An example of a youngstock vaccination plan on a dairy cattle farm [6]

\begin{tabular}{|l|l|}
\hline Age in days & Type of vaccine \\
\hline 15 & Pasteurellosis \\
\hline 30 & Clostridium enterotoxaemia \\
\hline 45 & Pasteurellosis \\
\hline 60 & Clostridium enterotoxaemia \\
\hline 75 & Anti-parasiticum \\
\hline 90 & Anti-parasiticum \\
\hline 120 & Inf. Bovine Respir. Disease (Herpes), Parainfluenza 3 and BVD \\
\hline 150 & Inf. Bovine Respir. Disease (Herpes), Parainfluenza 3 and BVD \\
\hline 180 & Brucellosis \\
\hline 195 & Brucellosis \\
\hline
\end{tabular}

Table 2. A short overview of risk factors associated with BVD in dairy cattle Risk factors associated with BVD occurrence

\begin{tabular}{|l|l|}
\hline New cattle is entering the farm & Calving of a cow \\
\hline No quarantine facilities are present & Sick animal pen \\
\hline No prior BVD-testing is conducted & Waste milk fed to calves \\
\hline Live contacts (wildlife) are occurring & People, cars/trucks, equipment, tools \\
\hline
\end{tabular}

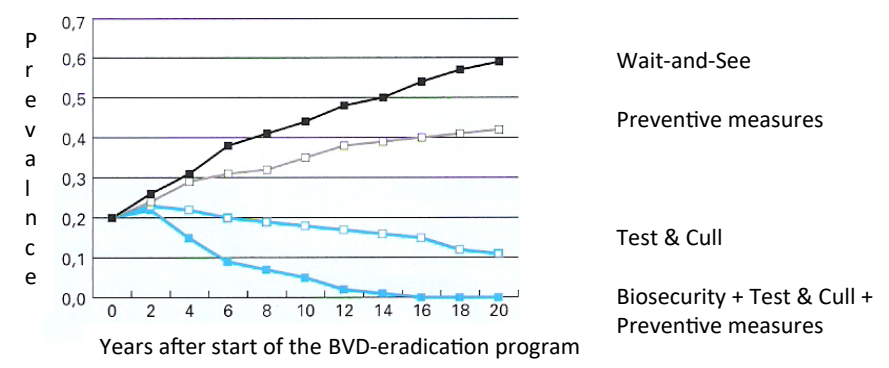

Figure 1. Example of results of different risk management strategies for combatting Bovine Virus Diarrhea at regional level [6]

latter statement is presented in Figure 1. This Figure deals with the results of different strategies for combatting Bovine Virus Diarrhea (BVD), caused by the BVD-virus [5]. The strategies are: Wait-and-See; Preventive measures; Test \& Cull; Biosecurity and Preventive measures combined with Test \& Cull $[1,2]$.

From the graph in Figure 1 one can read that the infectious disease BVD can be eradicated by combining different strategies and even without vaccination.
Risk identification and risk management can be applied at the level of an individual farm, but also at regional level and even nation-wide. The latter often relates to trade barriers when a certain country does not wish to import dairy products or live cattle from a country with a BVDprevalence. Several European countries have successfully implemented nation-wide BVD-control and monitoring programs for that reason. These programs are usually compulsory for dairy farmers.

\section{Concluding remarks}

The potential role and power of risk identification and risk management in disease control is largely underestimated in dairy veterinary practice. One reason is maybe that it is so easy to apply a vaccine just by injection. One nearly does not need to think, while in risk management one needs to think.

The domains of vaccination and risk management are complementary and therefore should need more attention and implementation.

Currently, the (veterinary) literature shows ample publications about risk identification and management. One should benefit from these.

The current COVID-19 situation points to the absolute need of combining the two forenamed domains. This is even more so, when vaccines are not (sufficiently) available and strong emphasis has to be put on risk management. Public health authorities should be much better prepared for this (including emergency risk communication).

\section{References}

1. Noordhuizen J, Wentink H (2001) Epidemiology: Developments in veterinary herd health programmes on dairy farms - a review. Vet $Q 23: 162-169$ [Crossref]

2. Noordhuizen J (2012) Dairy herd health and management. Context Products, U.K.

3. Kleen JL, Rehage J (2008) Communication skills in veterinary medicine. Tierarztliche Praxis 36: 5.

4. Kleen JL, Atkinson O, Noordhuizen, JPTM (2011) Communication in production animal medicine - modelling a complex interaction with the example of dairy herd health medicine. Irish Vet J 64: 8. [Crossref]

5. Viet AF, Fourichon C, Seegers H (2007) Review and critical discussion of assumptions and modelling options to study the spread of BVD-virus within a cattle herd. Epidemiol Infect 135: 706-721. [Crossref]

6. Noordhuizen J (2018) Report on a Dairy Farm Audit for improving heat stress management. (Unpublished data).

Copyright: (C2021 Noordhuizen J. This is an open-access article distributed under the terms of the Creative Commons Attribution License, which permits unrestricted use, distribution, and reproduction in any medium, provided the original author and source are credited. 